\title{
A Estimação das importações brasileiras de leite, 1991 a 2003
}

\author{
Dione Fraga dos Santos ${ }^{1}$ \\ Geraldo Sant'Ana de Camargo Barros ${ }^{2}$
}

Resumo: Com o intuito de avaliar o efeito das importações de lácteos sobre algumas variáveis macroeconômicas: quantidade importada, preço de importação, preço interno, PIB (Produto Interno Bruto) real e taxa de câmbio real foi especificado um modelo econométrico para a demanda de importação para o período de janeiro de 1991 a dezembro de 2003. A metodologia empregada para medir os impactos das importações de leite nas variáveis macroeconômicas foi a de séries temporais. Incorporou-se uma análise gráfica a partir do diagrama back to back analisado por Barros (2003). E, ainda, utilizou-se a equação de excesso de demanda para definir a demanda por importação de leite.

Palavras-chave: séries temporais, diagrama "back to back”, importação, leite

Classificação JEL: C32, F31

Abstract: In order to measure the impact of milk imports upon some macroeconomic variables such as imported quantity and price, domestic price, real gross domestic product (GDP) and exchange rate we specify

${ }^{1}$ Doutora em Economia Aplicada - Esalq-USP. Prof ${ }^{a}$ Adjunta I FEA-UFJF. dione.fraga@terra.com.br

${ }^{2}$ Pós-Doctor em Economia - University of Minnesota, Profo ${ }^{\circ}$ Titular Esalq-USP.

gscbarro@esalq.usp.br 
an econometric model for the import demand for the period january 1991 to december 2003. We implement the model using time series framework. We also analyse the market for milk imports through "back to back"graphic approach. Furthemore we also use te excess demand equation to define the demand for milk imports.

Key words: time series, back to back diagram, import, milk.

\section{JEL Classification: C32, F31}

\section{Introdução}

Na década de 1990 houve alterações significativas no setor agroindustrial do leite no país. Dentre elas destacam-se: i) a desregulamentação do rigoroso controle estatal após 46 anos (1945-1991); ii) o lançamento de novos produtos; iii) a entrada de multinacionais; iv) a criação do Mercado Comum do Cone Sul, e v) a abertura comercial generalizada.

O sistema agroindustrial do leite no país passou por importantes alterações desde o início da década de 1990. O setor parece ter reagido positivamente: houve reorganização intensa na pecuária leiteira que refletiu no aumento de competitividade para enfrentar a concorrência externa. O segmento de leite fluido experimentou a desregulamentação do setor após 46 anos de controle governamental no estabelecimento de preço do leite tipo C. Concomitantemente, houve lançamentos de novos produtos e derivados, a abertura econômica do país e a formação do Mercosul.

A abertura econômica e a formação do Mercosul levaram as empresas à competição com empresas de grande porte e a enfrentar os preços praticados muitas vezes distorcidos no resto do mundo. No mercado interno houve um processo de concentração das empresas de laticínios e a entrada de empresas multinacionais no mercado. Em paralelo, houve um aumento da participação relativa do leite UHT no mercado nacional de leite fluido durante a década de 1990.

Já o comércio mundial de lácteos tem a participação de poucos países, sendo os principais importadores e exportadores os países 
membros da Organização para a Cooperação e Desenvolvimento Econômico $\left(\mathrm{OCDE}^{3}\right)$. Países em desenvolvimento, como Índia e Brasil, possuem parcelas pouco expressivas do volume de comércio, apesar de serem o segundo e sexto produtores mundiais, respectivamente, em 2002.

No mercado externo, aumentou a importação brasileira de lácteos oriundos da Argentina, Uruguai, União Européia e Nova Zelândia, países que possuem preços abaixo da média dos praticados no mercado internacional provavelmente influenciados por medidas de dumping durante a década de 1990. Essa possibilidade de importações, segundo Barros et al. (2001), passou a estabelecer um limite superior de preços no segmento de leite brasileiro.

Como objetivo geral do presente artigo é enfocar o comércio internacional de leite no mundo destacando seus maiores exportadores e importadores e analisar, com base num modelo teórico e empírico, o comportamento das quantidades importadas de lácteos frente ao comportamento dos preços de importação, taxa de câmbio real, renda per capita, preço doméstico de leite, estabilização monetária e política anti-dumping.

A análise da demanda de importação desenvolvida neste trabalho incorpora variáveis macroeconômicas importantes, como a taxa de câmbio real, sendo a estimação feita através de procedimentos econométricos pertinentes considerando as questões de estacionariedade, co-integração e exogeneidade.

Ressalta-se que a contribuição dele é a investigação a respeito do efeito das variáveis macroeconômicas e do método adequado de estimação da função de demanda de importação de leite para o Brasil. O presente artigo está dividido em quatro itens sem contar com essa introdução. O primeiro refere-se à Metodologia. O segundo, aos Resultados e o próximo, à Conclusão. O último, à Bibliografia.

\footnotetext{
${ }^{3}$ Alemanha, Dinamarca, França, Itália, Noruega, Suíça, Canadá, Austrália, Áustria, Finlândia, Grã-Bretanha, Holanda, Portugal, Turquia, Espanha, Nova Zelândia, Bélgica, Irlanda, Grécia, Islândia, Luxemburgo, Suécia, Estados Unidos, Japão, México, República Tcheca, Eslováquia, Polônia e Coréia.
} 


\section{Metodologia}

\section{O Diagrama "back to back": fundamentação gráfica do modelo}

O modelo econômico admite uma sustentação gráfica por meio do diagrama back to back analisado por Barros (2003). Na Figura 1, tem-se o diagrama em que à direita do eixo vertical estão as curvas nominais de oferta e da demanda para o país $a$. À esquerda, as curvas do país $b$ são invertidas, pois as quantidades para essa região são obtidas da direita para a esquerda a partir do ponto de origem (O).

As curvas de excesso de oferta para os países são traçadas no intuito de mostrar os diferentes níveis de preços e seus respectivos montantes pelos quais a oferta excede a demanda. Obtém-se o excesso de oferta $\mathrm{ES}_{\mathrm{a}}$ e $\mathrm{ES}_{\mathrm{b}}$, tomando-se para cada nível de preço, a diferença horizontal entre a curva de oferta e a curva de demanda dos países.

Figura 1 - Diagrama back to back

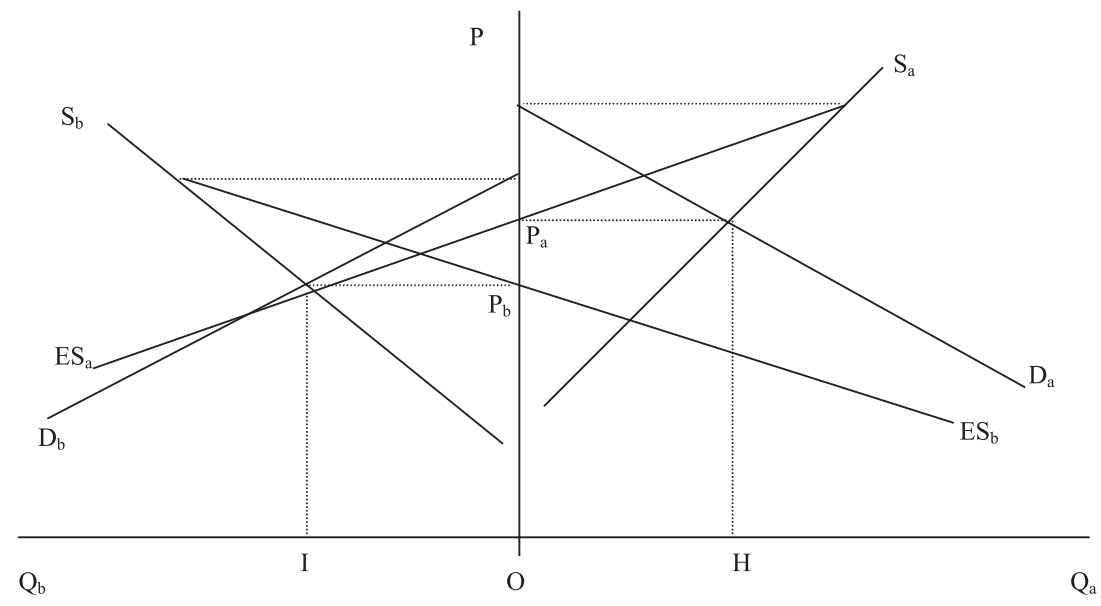

Fonte: Barros (2003)

Para referendar o modelo econômico proposto no trabalho, é necessário proceder algumas modificações no diagrama back to back apresentadas na Figura 1. Na Figura 2 observa-se que o país a representa o Brasil e o país $b$, o Resto do Mundo, a mercadoria em questão é o leite 
e ainda supondo: i) que o excesso de oferta no Brasil (ES $)$ representa o mesmo que um excesso de demanda, dado preço interno, $\mathrm{P}_{\mathrm{a}}^{0}$, superior ao preço internacional e de equilíbrio (em moeda nacional), $\mathrm{P}_{\mathrm{RM}}^{0}$, assim

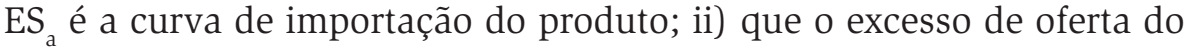
Resto do Mundo é infinitamente elástica, que representa a oferta de exportação do produto.

Figura 2 - Diagrama back to back com custo de transferência

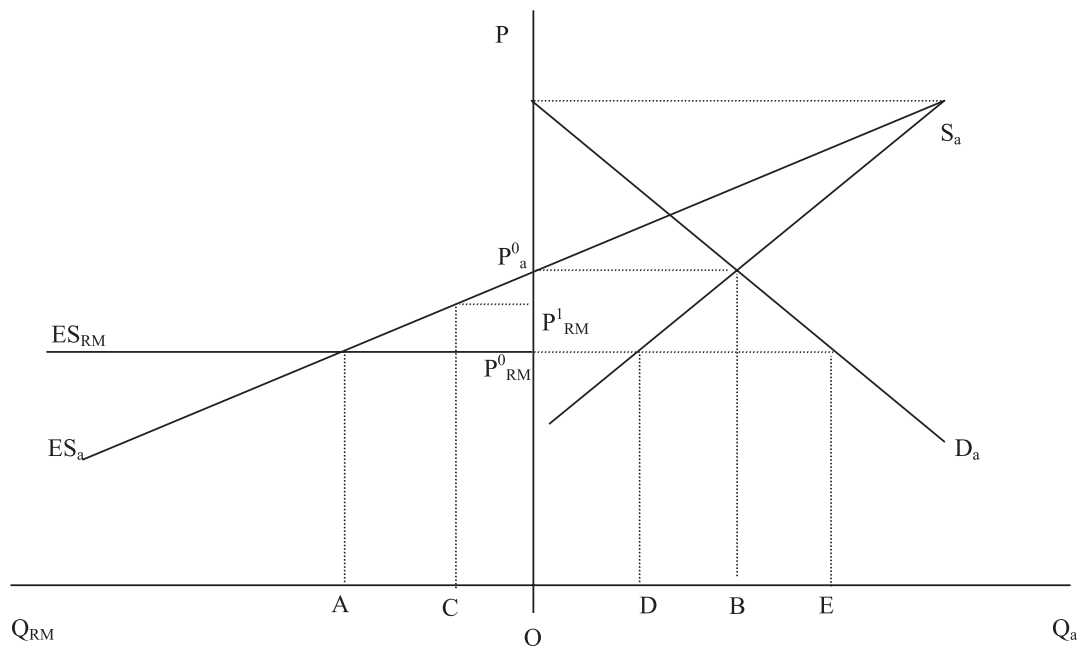

Fonte: Elaboração dos autores

Supondo inicialmente que o custo de transferência (CT) seja maior que a diferença de preços $\left(\mathrm{P}_{\mathrm{a}}^{0}-\mathrm{P}_{\mathrm{RM}}^{0}\right)$ em que CT inclui transporte, tarifa e outros, então, não haverá comércio de leite do Resto do Mundo para o Brasil. Para o estabelecimento do comércio, considera-se que o custo de transferência seja nulo $(\mathrm{CT}=0)$. O preço nas duas regiões se estabelece quando $\mathrm{ES}_{\mathrm{a}}=\mathrm{ES}_{\mathrm{RM}}$. $\mathrm{O} \mathrm{P}_{\mathrm{RM}}^{0}$ é o preço no qual o leite será comercializado da região chamada, Resto do Mundo para o Brasil. Em $\mathrm{P}_{\mathrm{RM}}^{0}$, haverá demanda por importação no Brasil pela quantidade DE, o mesmo montante de excesso de oferta do Resto do Mundo OA. Quando o custo de transferência CT $=\mathrm{k}$, então o preço no Brasil passará a ser $\mathrm{P}_{\mathrm{RM}}^{1}=\left(\mathrm{P}_{\mathrm{RM}}^{0}+\mathrm{k}\right)$, sendo que a quantidade importada pelo Brasil iguala-se a OC. Se ocorrer um aumento de renda interna no 
Brasil isso possibilitará um acréscimo na demanda que se desloca de $\mathrm{D}_{\text {a }}$ para D' na Figura 3. Com a nova curva de demanda por leite, D' haverá um aumento do preço interno de $\mathrm{P}_{\mathrm{a}}^{0}$ para $\mathrm{P}^{1}{ }_{\mathrm{a}}$ sem importação $\left(\mathrm{CT}>\mathrm{P}^{1}{ }_{\mathrm{a}}-\mathrm{P}_{\mathrm{RM}}^{0}\right)$. Com a alteração de preço, a curva de importação do Brasil desloca-se de ES a para ES' ${ }_{\mathrm{a}}$. A produção e o consumo nacionais serão iguais a OC a esse novo preço. Porém, o país importará a parcela $\mathrm{BE}$ oferecida ao preço $\mathrm{P}_{\mathrm{RM}}^{0}$ pelo Resto do Mundo se $\mathrm{CT}=$ 0 . Deve-se observar que a quantidade AO exportada pelo Resto do Mundo é igual à quantidade $\mathrm{BD}$. E o montante a mais que se espera importar DE é igual à AF.

Figura 3 - Diagrama back to back com alteração da demanda

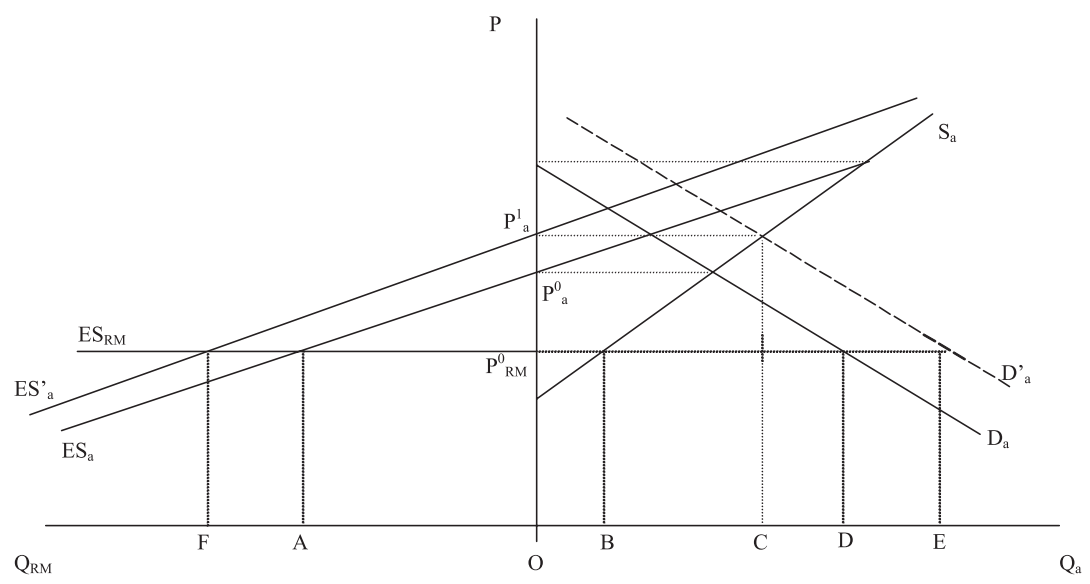

Fonte: Elaboração dos autores

\section{Especificação da equação de excesso de demanda}

As representações gráficas feitas no item anterior para a conceituação da curva de excesso de demanda admitem um tratamento matemático para melhor definir o modelo a ser estimado no presente estudo. Vale destacar, parte da definição da função de excesso de demanda definida matematicamente por:

$$
\begin{aligned}
M^{d} & =Q^{d}-Q^{s} \\
M^{d} & =Q^{d}\left(P_{d}, P_{m}, E, Y\right)-Q^{s}\left(P_{d}, W\right)
\end{aligned}
$$


linearizada leva a estimação da função de demanda por leite importado proposta:

$$
\begin{aligned}
& M^{d}=\left(\alpha_{0}+\alpha_{1} P_{d}+\alpha_{2} P_{m}+\alpha_{3} E+\alpha_{4} Y\right)-\left(\beta_{0}+\beta_{1} P_{d}+\beta_{3} W\right) \\
& M^{d}=\left(\alpha_{0}-\beta_{0}\right)+\left(\alpha_{1}-\beta_{1}\right) P_{d}+\alpha_{2} P_{m}+\alpha_{3} E+\alpha_{4} Y-\beta_{3} W
\end{aligned}
$$

Em que:

$M^{d}=$ função de excesso de demanda por leite importado; $Q^{d}=$ função de demanda por leite importado; $Q^{s}=$ função de oferta de leite; $P_{d}$ $=$ preço do leite no mercado doméstico; $P_{m}=$ preço do leite no mercado externo; $E=$ taxa de câmbio; $Y=$ variáveis deslocadoras da demanda (renda interna, gosto e preferência); $W=$ variáveis deslocadoras da oferta (safra, entressafra, melhoria genética); $\alpha_{0}, \alpha_{1}, \alpha_{2}, \alpha_{3}, \alpha_{4}, \beta_{0}, \beta_{1}, \beta_{3}$ $=$ coeficientes associados às variáveis explicativas.

Pode-se ainda, como forma de adequar o modelo proposto na equação 1 com os objetivos do trabalho, adicionar algumas variáveis qualitativas do tipo dummy. O objetivo é admitir que existam outras variáveis importantes na especificação da demanda por leite importado que não foram incorporadas na equação (1), como por exemplo, o efeito da estabilização monetária com o advento do Plano Real em julho de 1994 e o efeito das medidas anti-dumping que o governo implementou a partir de 1999. Matematicamente, tem-se:

$$
M^{d}=\left(\alpha_{0}-\beta_{0}\right)+\left(\alpha_{1}-\beta_{1}\right) P_{d}+\alpha_{2} P_{m}+\alpha_{3} E+\alpha_{4} Y-\beta_{3} W+\gamma_{1} \mathrm{D}_{1}+\gamma_{2} \mathrm{D}_{2}
$$

Em que: $\mathrm{D}_{1}=$ dummy para a estabilização monetária; $\mathrm{D}_{2}=$ dummy para as medidas anti-dumping; $\gamma_{1}, \gamma_{2}=$ coeficientes associados às variáveis dummies.

Será feito o teste de causalidade de Granger para a relação entre $M^{d}$ e $P_{m}$. Lembre-se que a formulação gráfica e algébrica adotada necessita da hipótese de que $P_{m}$ seja exógena em relação à $M^{d}$. Ou seja, $M^{d}$ não afeta $P_{m}$ contemporaneamente (por pressuposição) ou com defasagens. Caso esta relação se mostre bicausal, não se sustenta a exogeneidade em vista de efeito defasado de $M^{d}$ sobre $P_{m}$. Deve-se abrir, então, a possibilidade de ocorrer também efeito contemporâneo entre essas variáveis. Segundo Barros (2003), há que se substituir, portanto, a linha horizontal de $\mathrm{ES}_{\mathrm{RM}}$ por uma linha negativamente inclinada: maiores pre- 
ços aumentam o excesso de oferta (medido da esquerda para a direita) mundial para o Brasil. Essa linha pode ser representada com $P_{m}$ como variável dependente e $M^{d}$ como variável explicativa.

$$
P_{m}=\lambda_{0}+\lambda_{1} M^{s}+\lambda_{2} L^{w}+v_{t}
$$

Em que $M^{s}=$ oferta do Resto do Mundo para o Brasil, $L^{w}=$ total de exportações mundiais de leite e $\lambda_{1}<0$ e $\lambda_{2}>0$. O modelo se equilibra quando $M^{d}=M^{s}=M$

Conforme Gujarati (1995), caso exista a simultaneidade, os métodos de Mínimos Quadrados de Dois Estágios (MQ2E) e de variáveis instrumentais serão os indicados, pois seus estimadores serão consistentes e eficientes.

Para se testar a exogeneidade fraca far-se-á o teste de Hausman citado em Gujarati (1995). Ou seja, verificar-se-á se o resíduo da equação (2) é significativo pelo teste $t$ de Student na equação (3). Da mesma forma, se o resíduo da equação (3) for significativo pelo teste $t$ na equação (2) existirá causalidade fraca, condição suficiente para que exista causalidade entre a variável $M^{d}$ e Pm (Greene, 2000; Judge et alii, 1985).

\section{Modelo empírico}

Para analisar empiricamente os determinantes das importações de leite pelo Brasil foi estimada a função de demanda por leite importado. A equação genérica de importação de leite para o Brasil é apresentada pela equação (3):

$$
\begin{aligned}
& M^{d}=\left(\alpha_{0}-\beta_{0}\right)+\left(\alpha_{1}-\beta_{1}\right) P_{d}+\alpha_{2} P_{m}+\alpha_{3} E+\alpha_{4} Y+\gamma_{1} \mathrm{D}_{1}+\gamma_{2} \mathrm{D}_{2}+\varepsilon_{\mathrm{t}} \\
& M^{d}=\theta_{0}+\theta_{1} P_{d t}+\alpha_{2} P_{m t}+\alpha_{3} E_{t}+\alpha_{4} Y_{t}+\gamma_{1} \mathrm{D}_{1}+\gamma_{2} \mathrm{D}_{2}+\varepsilon_{t}
\end{aligned}
$$

Em que: $M_{t}=$ quantidades mensais de importação de lácteos no período de 1991 a 2003; $\theta_{0}=$ coeficiente autônomo; $\theta_{1}, \alpha_{2}, \alpha_{3}, \alpha_{4}, \beta_{1}, \gamma_{1}, \gamma_{2}$ $=$ coeficientes associados às variáveis explicativas; $P_{d t}=$ preços mensais de leite em R \$/1000 litros no período de 1991 a 2003; $P_{m t}=$ preços mensais de importação de lácteos em US\$/1000 litros no período de 1991 a 2003; $E_{t}=$ taxa mensal de câmbio real no período de 1991 a 2003; $\mathrm{Y}_{\mathrm{t}}$ $=$ produto interno mensal per capita deflacionado pelo IGP-DI (FGV) no 
período de 1991 a 2003; $D_{1}=$ estabilização monetária no mês de julho de 1994 (0 até junho de 1994 e 1 nos demais meses de 1994); $D_{2}=$ medidas anti-dumping a partir de janeiro de 1999 (0 até dezembro de 1998 e 1 nos demais meses); $\varepsilon_{t}=$ erro aleatório no período analisado.

Os sinais esperados para os parâmetros da equação (3) são $\theta_{0}>0, \theta_{1}>0, \alpha_{2}<0, \alpha_{3}<0, \alpha_{4}>0, \gamma_{1}<0, \gamma_{2}>0$. A literatura consultada em vários trabalhos sobre estimação da equação de demanda de importação sugere que se aplique na equação (3) o modelo duplo-logaritmo. Procurar-se-á determinar por meio do teste de sentido de causalidade de Granger, se a quantidade importada de leite $\left(M_{t}^{d}\right)$ é causada pelo preço de importação $\left(P m_{t}\right)$, vice-versa ou em ambos os sentidos. E, determinar se a exogeneidade é fraca por meio do teste de Hausman. Além disso, procede-se a uma série de testes econométricos como de raiz unitária e o de co-integração de Johansen (Ver mais detalhes em Guajarati, 1995 e Bacchi, 2001).

\section{Resultados e discussão}

Os resultados dos testes de raiz unitária para as séries foram tomados nos logaritmos: importação mensal de leite $\left(l M_{t}^{d}\right)$, preço mensal de leite no mercado interno $\left(l P_{d t}\right)$, preço mensal de importação de leite $\left(l P_{m t}\right)$, taxa mensal de câmbio real $\left(l E_{t}\right)$, PIB mensal per capita $\left(l Y_{t}\right)$ e exportação mensal mundial de leite $\left(l L^{w}\right)$. Utilizou-se o procedimento proposto por Enders (2004) para identificar o modelo específico a ser considerado no teste, incluindo ou não termos deterministas.

As variáveis relacionadas pelo modelo apresentaram no teste de raiz unitária ordem de integração igual a 1, [I(1)]. Isso significa que elas não rejeitam a hipótese nula de raiz unitária, sendo necessário que na especificação do modelo elas sejam consideradas nas diferenças de primeira ordem. Ou seja, a estacionariedade das séries só foi configurada nas diferenças, quando os valores encontrados foram superiores ao valor crítico em nível de 1\% (Tabela 1).

Para todas as variáveis foram encontrados níveis de significância da estatística Q do teste de Ljung-Box maiores que 0,48, rejeitando a hipótese nula desse teste, que considera a existência de autocorrelação serial entre os resíduos. 
Tabela 1. Teste de raiz unitária em modelo sem constante e sem tendência, estatística $\tau^{1}$

\begin{tabular}{l|c|c||c}
\hline \multicolumn{2}{c}{$\Delta x_{t}=\sigma_{1} \Delta x_{t-1}+\sum_{j=1}^{n} \sigma_{j} \Delta x_{t-j}+\varepsilon_{t}$} & $\mathrm{H}_{0:} \sigma_{1}=0$ \\
\hline \multicolumn{1}{c}{ Variáveis } & Valor de $p-1$ & $\begin{array}{c}\text { Valor da estatística t } \\
\mathrm{H}_{0}: \sigma_{1}=0\end{array}$ & $\begin{array}{c}\text { Nível de significância da } \\
\text { estatística "Q" }\end{array}$ \\
$\Delta l M_{t}^{d}$ & 12 & $-3,69^{*}$ & 0,99 \\
$\Delta l P_{d t}$ & 6 & $-7,29^{*}$ & 0,62 \\
$\Delta l P_{m t}$ & 8 & $-4,86^{*}$ & 0,48 \\
$\Delta l E_{t}^{(2)}$ & 1 & $-5,20^{*}$ & 0,89 \\
$\Delta l Y_{t}$ & 12 & $-3,35^{*}$ & 0,49 \\
$\Delta l L_{t}^{w}$ & 12 & $-3,18^{*}$ & 0,80 \\
\hline
\end{tabular}

Fonte: Dados da pesquisa.

(1) Valores críticos de $\tau$ conforme descrito por Dickey e Fuller (1981) e corresponde a -1,95 em nível de $5 \%$ e $-2,60$ em nível de $1 \%$.

(2) Ajustado com duas quebras estruturais (janeiro de 1999 e maio de 2002).

Significativo ao nível de significância de $1 \%$.

Os testes de co-integração foram realizados entre as variáveis duas a duas, para obter os termos de correção de erro para os testes de causalidade. Os resultados estão apresentados na Tabela 2. O valor do teste “t” para o resíduo $(-3,83)$ no teste de co-integração é maior, em módulo, que o valor crítico (vc) de -3,55 em nível de significância de $10 \%$. Conclui-se que há um vetor de co-integração entre as variáveis da equação (i). Considerando a equação (ii), o valor do teste $t$ para o resíduo $(-3,85)$ no teste de co-integração é significativo ao nível de $10 \%$. Conclui-se que na equação (ii) existe um vetor de co-integração.

Os resultados das equações definidas no teste de causalidade ${ }^{4}$ são apresentados na Tabela 3. As relações de causalidade fornecem uma indicação mais precisa das relações comportamentais do mercado importador de leite no país.

${ }^{4}$ As defasagens do teste de causalidade foram obtidas pelo critério de Akaike e Schwartz. 
Tabela 2. Resultados do teste de co-integração

\begin{tabular}{ccc}
\hline $\begin{array}{c}\text { Regressão estimada para testar a co- } \\
\text { integração }\end{array}$ & $\begin{array}{c}\text { Resultado do teste de estacionariedade } \\
\text { aplicado no resíduo estimado }\end{array}$ \\
(i) $l M_{t}^{d}=8,007548-0,5634245 l P_{m t}$ & $\mathrm{t}=-3,83$ \\
$\mathrm{R}^{2}=0,99$ & $\mathrm{Q}(\%)=0,969$ & $\begin{array}{l}\mathrm{vc}(5 \%)=-3,89^{*} \\
\mathrm{vc}(10 \%)=-3,55^{*}\end{array}$ \\
(ii) $l P_{m t}=5,120586-0,0180994 l M_{t}^{d}$ & $\mathrm{t}=-3,85$ \\
$\mathrm{R}^{2}=0,99$ & $\mathrm{Q}(\%)=0,847$ & $\begin{array}{l}\mathrm{vc}(5 \%)=-3,89^{*} \\
\mathrm{vc}(10 \%)=-3,55^{*}\end{array}$ \\
\hline
\end{tabular}

* valor crítico para 100 observações e 1 variável (Harris, 1995)

Observa-se um sentido bi-causal no mercado importador de leite, em que a quantidade importada de leite $\left(\Delta l M_{t}^{d}\right)$ causa o preço de importação de leite $\left(\Delta l P_{m t}\right)$ e, simultaneamente, o preço de importação de leite $\left(\Delta l P_{m t}\right)$ causa a quantidade de leite importado $\left(\Delta l M_{t}^{d}\right)$. Portanto, será estimado o modelo pelo método de Mínimos Quadrados de Dois Estágios (MQ2E), uma vez que existe uma relação endógena no sistema de equações. Além disso, as equações (2) e (3) são sobre-identificadas indicando a utilização do método de MQ2E.

Tabela 3. Resultados do teste de causalidade de Granger com termo de correção de erro

\begin{tabular}{llccc}
\hline Variável dependente & Variável explicativa & $p$ Teste $\mathrm{F}$ & $p$ Teste $\mathrm{t}$ & Teste Q \\
\hline$\Delta l M_{t}^{d}$ & $\Delta l P_{m t}$ & 0,001 & 0,003 & 0,16 \\
$\Delta l P_{m t}$ & $\Delta l M_{t}^{d}$ & 0,001 & 0,002 & 0,18 \\
\hline
\end{tabular}

Fonte: Dados de pesquisa

Conclui-se que há exogeneidade fraca, pois os resíduos são significativos pelo teste $t$. Assim, pode-se afirmar que existe causalidade entre $\Delta l M_{t}^{d}$ e $\Delta l P_{m t}$, sendo condição necessária à exogeneidade forte. A fraca, referendada pelo teste de Hausman, é condição suficiente para confirmar a causalidade no sentido de Granger, uma vez que o objetivo é a estimação de variáveis endógenas. Ver Tabela 4. 
Nos resultados da análise de co-integração para as séries de mesma ordem, os testes do máximo autovalor e do traço indicam que há dois vetores de co-integração (Tabela 5). Dessa maneira, os resultados mostram que existem relações de equilíbrio de longo prazo entre as variáveis.

Tabela 4. Resultados do teste de Hausman

\begin{tabular}{lcccc}
\hline \multirow{2}{*}{ Variáveis } & \multicolumn{2}{c}{$\Delta l M_{t}^{d}$} & \multicolumn{2}{c}{$\Delta l P_{m t}$} \\
\cline { 2 - 5 } & Coeficiente & Valor $p$ de $t$ & Coeficiente & Valor $p$ de $t$ \\
\hline Constante & 9,045811 & 14,96971 & 4,342549 & 24,73361 \\
$\Delta l M_{t}^{d}$ & - & - & $-0,652837$ & $-40,32745$ \\
$\Delta l P_{d t}^{(1)}$ & 2,488259 & 7,84625 & - & - \\
$\Delta l P_{m t}$ & $-6,350305$ & $-31,97191$ & - & - \\
$\Delta l E_{t}{ }^{(1,2)}$ & $-1,358041$ & $-5,70965$ & $-0,858267$ & $-13,43305$ \\
$\Delta l Y_{t}^{(1)}$ & 6,410267 & 9,46624 & 1,823598 & 11,91541 \\
$\Delta l L_{t}^{w}(1)$ & 1,328282 & 3,40598 & $-0,611759$ & $-7,17624$ \\
$\hat{\mathrm{\imath}}_{t}$ & $6,331356 *$ & 27,72871 & - & - \\
$\hat{\varpi}_{t}$ & - & - & $0,652837 *$ & 35,75900 \\
$\mathrm{r}^{(1)}$ & $-0,024938$ & $-15,31705$ & $-0,006616$ & $-18,19791$ \\
$\mathrm{r}^{(1)}$ & $-0,184431$ & $-14,06500$ & $-0,093189$ & $-24,29216$ \\
$\mathrm{D}_{1}$ & $-0,195657$ & $-5,28205$ & 0,001113 & 0,12177 \\
$\mathrm{D}_{2}$ & $-0,004756$ & $-0,20205$ & 0,054068 & 8,88968 \\
& \multicolumn{5}{c}{$\mathrm{F}=56,12^{*}$} & \multicolumn{3}{c}{$\mathrm{F}=89,47^{*}$} \\
\hline
\end{tabular}

Fonte: Dados da pesquisa.

* Significativo ao nível de significância de $1 \%$.

(1) Variável defasada; ${ }^{(1,2)}$ Variável defasada com duas quebras estruturais.

\section{Estimação das funções de demanda e preço de leite importado}

Na estimação das referidas equações (Tabela 6), todas as variáveis apresentaram sinais coerentes com a teoria econômica e nível de significância a pelo menos $10 \%$ à exceção das variáveis dummies que indicavam estabilidade econômica (D1) e política anti-dumping (D2) 
relacionada na equação de demanda por leite importado $\left(\Delta l M_{t}^{d}\right)$. As variáveis relacionadas pela pesquisa do comportamento da demanda por leite importado explicaram $34 \%$ das importações de leite conforme sugere o coeficiente de determinação.

Tabela 5. Resultados do teste de co-integração de Johansen

\begin{tabular}{cccc}
\hline Hipótese Nula & Hipótese Alternativa & $\lambda_{\max }$ & $\lambda_{\text {trace }}$ \\
\hline $\mathrm{r} \leq 5$ & $\mathrm{r}=6$ & 3,628 & 3,628 \\
$\mathrm{r} \leq 4$ & $\mathrm{r}=5$ & 10,814 & 14,441 \\
$\mathrm{r} \leq 3$ & $\mathrm{r}=4$ & 15,053 & 29,494 \\
$\mathrm{r} \leq 2$ & $\mathrm{r}=3$ & 26,845 & 56,339 \\
$\mathrm{r} \leq 1$ & $\mathrm{r}=2$ & $44,088^{*}$ & $100,427^{*}$ \\
$\mathrm{r} \leq 0$ & $\mathrm{r}=1$ & $86,955^{*}$ & $187,382^{*}$ \\
\hline
\end{tabular}

Fonte: Dados da pesquisa.

* Significativo a 1\% de significância [valores críticos em Osterwald-Lenum (1992)].

As variáveis relacionadas para a estimação da equação de preço de leite importado $\left(\Delta l P_{m t}\right)$ apresentaram sinal coerente com a teoria econômica. As variáveis, quantidade demandada de leite pelo país e produção mundial de leite explicaram $22 \%$ dos preços de importação pagos pelo Brasil como sugere o coeficiente de determinação.

Para interpretar os resultados, tomou-se como ilustração o efeito do crescimento da economia brasileira. Um aumento no PIB brasileiro tende a aumentar o volume importado que, por sua vez eleva o preço de importação, contendo parcialmente o efeito de crescimento das importações. Da mesma forma, uma expansão da produção mundial reduz o preço de importação, que provoca um aumento nas importações, que atenua a queda original de preço.

Tratando-se de um sistema de duas equações simultâneas, a interpretação dos efeitos de choques nas variáveis exógenas sobre as endógenas $\left(\Delta l M_{t}^{d}\right.$ e $\left.\Delta l P_{m t}\right)$ ficou facilitada a partir de sua forma reduzida. Esta pode ser obtida resolvendo-se o sistema para as variáveis endógenas em termos de todas as exógenas. Por exemplo, pode-se substituir $\Delta l M_{t}^{d}$ na equação de $\Delta l P_{m t}$; o resultado foi então substituído em $\Delta l M_{t}^{d}$. Os resultados estão na Tabela 7 . 
Tabela 6. Estimativas do ajustamento em dois estágios da demanda por leite importado $\left(M_{t}^{d}\right)$ e do preço de importação $\left(P_{m t}\right)$

\begin{tabular}{lcc}
\hline \multirow{2}{*}{ Variáveis } & $\Delta l M_{t}^{d}$ & $\Delta l P_{m t}$ \\
\cline { 2 - 3 } & & Coeficientes \\
\hline Constante & $6,185614^{* *}$ & $0,645634^{* *}$ \\
$\Delta l P_{m t^{(1)}}$ & $-1,562731^{* *}$ & - \\
$\Delta l M_{t}^{d}$ & - & $-0,124643^{* *}$ \\
$\Delta l L_{t^{(1)}}^{w}$ & - & $-0,439108^{*}$ \\
$\Delta l E_{t^{(1,2)}}$ & - \\
$\Delta l Y_{t^{(1)}}$ & $-1,183131^{* *}$ & - \\
$\Delta l P_{d t^{(1)}}$ & $2,982639^{* *}$ & - \\
$r 1^{(1)}$ & $1,643824^{*}$ & $-0,002861^{* *}$ \\
$r 2^{(1)}$ & $-0,009389^{* *}$ & $-0,012235^{* *}$ \\
$D 1$ & $-0,132842^{* *}$ & - \\
$D 2$ & 0,001714 & - \\
$R^{2}$ & 0,074887 & 0,2224 \\
\hline
\end{tabular}

Fonte: Dados de pesquisa.

** Significativo a $1 \%$, * Significativo a $10 \%$.

(1) Variável defasada;

$(1,2)$ Variável defasada e com duas quebras estruturais.

Tabela 7. Resultados para a forma reduzida do sistema

\begin{tabular}{lcc}
\hline \multirow{2}{*}{ Variáveis } & $\Delta l M_{t}^{d}$ & $\Delta l P_{m t}$ \\
\cline { 2 - 3 } Constante & \multicolumn{2}{c}{ Coeficientes } \\
$\Delta l E_{t}$ & 6,429 & $-0,158$ \\
$\Delta l Y_{t}$ & $-1,469$ & 0,184 \\
$\Delta l P_{d t}$ & 3,706 & $-0,463$ \\
$\Delta l L_{t}^{w}$ & 2,042 & $-0,255$ \\
$r 1$ & $-0,852$ & 0,546 \\
$r 2$ & $-0,005$ & 0,001 \\
$D 1$ & $-0,068$ & 0,021 \\
$D 2$ & 0,002 & 0,003 \\
\hline
\end{tabular}

Fonte: Dados de pesquisa. 
Na equação da demanda por leite importado, as variáveis preço de importação de leite, taxa de câmbio real, PIB per capita real e preço interno de leite apresentaram coeficientes significativos e sinais coerentes com a teoria econômica. As elasticidades estimadas foram todas próximas ou superiores à unidade, revelando um mercado bastante dinâmico com ajustes rápidos no curto prazo. A demanda por importação mostrou-se bastante elástica tanto em relação ao preço de importação como ao preço doméstico. As elasticidades foram de magnitudes bem parecidas - mas, claro, de sinais opostos -, sugerindo uma boa integração do segmento leiteiro ao mercado internacional: variações iguais nos dois preços mantida a taxa de câmbio, pouco alterariam o volume importado de leite. O crescimento da renda tendeu a resultar em incremento importante das importações, indicando que o setor nacional tem dificuldade de atender a um crescimento mais rápido do consumo interno. O câmbio real foi um fator relevante para as importações de lácteos na medida que é determinante do preço relativo dos produtos nacional e importado.

No tocante à oferta de leite importado, nota-se que o preço pago pelo Brasil é negativamente influenciado pela oferta internacional. As importações brasileiras influenciam o preço pago em moeda estrangeira, mas o sinal negativo obtido contraria as expectativas teóricas, embora não seja impossível que tal sinal ocorra na prática: o preço pago caia com o volume de compras, como é comum em práticas comerciais. É importante também reconhecer que as dummies incluídas para captar as variações de políticas econômicas, como a estabilização monetária e as políticas anti-dumping, não apresentaram efeitos significativos. Como se supunha, é possível que seus efeitos tenham sido captados por outras variáveis presentes no modelo como preços e câmbio.

\section{Conclusões}

Vale destacar, na equação da demanda por leite importado, as variáveis preço de importação de leite, taxa de câmbio real, PIB per capita real e preço interno de leite por serem significativas e apresentarem sinais coerentes com a teoria econômica. As elasticidades estimadas foram todas próximas ou superiores à unidade, revelando um mercado bastante dinâmico com ajustes rápidos no curto prazo. A demanda 
por importação mostrou-se bastante elástica tanto em relação ao preço de importação como ao preço doméstico. As elasticidades foram de magnitudes bem parecidas, sugerindo uma boa integração do segmento leiteiro ao mercado internacional: variações iguais nos dois preços, mantida a taxa de câmbio, não alterariam o volume importado de leite. O crescimento da renda tende a resultar em incremento importante das importações, indicando que o setor nacional tem dificuldade de atender a um crescimento mais rápido do consumo interno. O câmbio real tem sido um fator relevante para as importações de lácteos na medida que é determinante do preço relativo dos produtos nacional e importado.

No tocante à oferta de leite importado, nota-se que as importações brasileiras influenciam o preço pago em moeda estrangeira. É importante também reconhecer que, as dummies incluídas para captar as variações de políticas econômicas, como a estabilização monetária e as políticas anti-dumping não apresentaram efeitos significativos. É possível que seus efeitos tenham sido captados por outras variáveis presentes no modelo. Os efeitos da estabilização monetária podem ter sido captados pela variável câmbio real, que se manteve fortemente valorizado no período que seguiu o Plano Real. O mesmo pode ter cumprido a variável representativa do PIB brasileiro.

\section{Referências bibliográficas}

BACCHI, M. R. P. Integração, co-integração e modelo de correção de erro: uma introdução. Piracicaba: Esalq-USP, 2001 (mimeo).

BARROS, G.S.C. Economia da Comercialização. Centro de Estudos Avançados em Economia Aplicada. ESALQ/USP, 1987, 360 p. http:// www.cepea.esalq.usp.br (Março de 2003) .

ENDERS, W. Applied econometric time series. New York: John Wiley \& Sons, 2004.

GREENE, W. H. Econometric Analysis. New Jersey: Prentice Hall, 2000.

GUJARATI, D. N. Basic Econometrics. Singapore: McGraw-Hill International Editions, 1995. 
HARRIS, R. Using cointegration analysis in econometric modelling. UK: Prentice Hall-Harvest Wheatsheaf, 1995.

JUDGE, G. G.; GRIFFITHS, W. E.; HILL, R. C.; LÜTKEPOHL, H.; LEE, T. C. The theory and practice of econometrics. New York: John Wiley and Sons, 1985.

OSTERWALD-LENUM, M. A note with quanlites of the asymptotic distribution of the maximun likehood cointegration rank test statistics. Oxford Bulletion of Economics and Statistics, v. 54, n. 3, 1992.

Recebido em novembro de 2005 e revisto em maio de 2006 